\title{
Extended PDMS stiffness range for flexible systems
}

\author{
R. Seghir ${ }^{\mathrm{a}}, \mathrm{S}$. Arscott $\mathrm{a}^{\mathrm{a}, *}$ \\ ${ }^{a}$ Institut d'Electronique, de Microélectronique et de Nanotechnologie (IEMN), CNRS UMR8520, University of Lille, Avenue Poincaré, Cité Scientifique, Villeneuve \\ d'Ascq 59652, France.
}

\begin{abstract}
The use of polymers in the context of flexible systems such as flexible sensors leads to an incompatibility issue: On one hand, the flexibilty of the polymer must not be to the detriment of the fabrication process, e.g. excessive thermal expansion leading to process failure and on the other hand, certain applications will require high flexibility and also a specific mechanical stiffness, e.g. artificial skin, smart clothes, flexible screen ... In other words, a compromise is necessary between rigidy for processing and controlled flexibility for applications. In this context it is crutial to be able to tune the mechanical properties of such polymers. Polydimethylsiloxane (PDMS) is a very versatile and useful soft polymeric material - Elastic modulus typically $\approx 1 \mathrm{MPa}$. This paper investigates the stiffness tunability of PDMS by varying the hardening agent to PDMS base ratio over 19:1 to 2:1, and using two extreme curing processes, i.e. 120 minutes at $100^{\circ} \mathrm{C}$ and 2 days at $165^{\circ} \mathrm{C}$. It was observed that the stiffness of PDMS can be accuratly controlled from $800 \mathrm{kPa}$ to $10 \mathrm{MPa}$ with a rupture limit higher than $20 \%$. To our knowlegde this is the highest reported elastic modulus in PDMS by combining mixing ratio and curing temperature. The impact of such a stiffness variation on potential functional properties such as the rupture limit, Poisson's ratio and material's wetting contact angle is also analysed. We observe that the wetting contact angle depends on the bulk mechanical properties of the PDMS. The observations will be of use to all technological communities who are engaged in using PDMS-type polymers for their specific applications.
\end{abstract}

Keywords: polydimethylsiloxane, process parameters, mechanical properties, mixing ratio, curing, wetting contact angle, rutpure

\section{Introduction}

Although the first organosilicon was first synthesized over one hundred years ago [1], it is only in the last 20 years that polymeric organosilicon compounds - such as polydimethylsiloxane (PDMS) - have found applications in areas such as flexible electronics [2, 3, 4, 5, 6], moulding and soft lithography [7, 8, 9, 10, 11, 12, 13, 14], microelectromechanical systems (MEMS) [15, 16, 17], self-healing materials [18], laboratoryon-a-chip and microfluidics [19, 20, 21, 22, 23]. The physical and chemical properties of PDMS, such as its usability over a wide temperature range $\left(\mathrm{T} \in[-100,200]^{\circ} \mathrm{C}\right)$, its flexibility (Elastic modulus $\mathrm{E} \approx 1 \mathrm{MPa}[24])$, its low chemical reactivity as well as its relativelly hydrophic behavior, its transparent nature, its biocompatibility [25], its low cost. . . have made it useful in such a wide range of application fields. In such a context a clear understanding of its mechanical behavior and its ability to reach specifics properties, for example a specific rigidity useful for a specific application can be of primary importance.

Much work has been done into the investigation of the mechanical properties of PDMS and their potential tunability [24, 26, 21, 27, 28, 16, 29, 30, 17, 31, 4, 32, 33, 34, 35]. The largest observed tensile stiffness, using 10:1 PDMS, to date is less than $4 \mathrm{MPa}$ [28] after 7 days post-baking at $100^{\circ} \mathrm{C}$ and less than $3 \mathrm{MPa}$ for post-baking at $200^{\circ} \mathrm{C}$ during 18 minutes [35]. It is known that the stiffness of PDMS can be tuned by modifying three main parameters: (1) the cross-linker agent concentration, (2) the curing temperature and (3) the curing time. Other parameters also affect stiffness, e.g. film thickness [30], micromachined object dimensions [31] and the loading strain rate [16]. Nervetheless the major part of these studies have focused only on a specific parameter at one time, frequently in a limited range and no work has been done to harden PDMS material beyond the classical rigidity range, i.e. [0.1 - 4] $\mathrm{MPa}$ [28], that would increase the applications spectrum of the material. As a consequence it remains very difficult to find a complete study where fabrication recipies and mechanical properties, over a large domain of applications, are brought together.

In this way, we propose here to investigate a large range of cross-linker agent concentrations, from $5 \%$ to $33 \%$, meaning mixing ratios by weight ranging from $19: 1$ to $2: 1$ and two curing processes, i.e. $2 \mathrm{~h}$ at $100^{\circ} \mathrm{C}$ and $48 \mathrm{~h}$ at $165^{\circ} \mathrm{C}$ including different curing methods: conventional oven and hot plate. In the first part of the paper, the fabrication process is presented, then uniaxial mechanical tests are detailed and elastic modulus, rupture strain as well as Poisson's ratio are presented. Finally the impact of the stiffness on the wetting contact angle is also discussed.

\footnotetext{
${ }^{*}$ Corresponding authors

Email addresses: rian.seghir@iemn. univ-lille1.fr (R. Seghir), steve.arscott@iemn.univ-lille1.fr (S. Arscott)
} 


\section{Material and methods}

\subsection{Fabrication}

All chemicals used in this work were used unmodified and off-the-shelf. All processing was performed in a class ISO 5/7 cleanroom. PDMS was purchased from Dow Corning Corporation, USA as a two liquid component kit, Sylgard ${ }^{\circledR} 184$ Elastomer, containing the vinyl-terminated base and the curing agent (methyl hydrogen siloxane). We define the PDMS mixtures as the ratio $\mathrm{A}: \mathrm{B}$ where $\mathrm{A}$ is the vinyl-terminated base weight content and B is the cross-linker agent weight content. PDMS mixtures were first prepared by mixing the base and the curing agent at the following mixing ratios by weight: 19:1, $10: 1,9: 1,8: 1,7: 1,4: 1$ and $2: 1$ in order to understand the mechanical behavior of low (5\%) to high (33\%) curing agent fraction. Note that the Sylgard ${ }^{\circledR} 184$ data-sheet [36] advises producing PDMS following a "by weight" ratio of 10:1 (i.e. $9 \%$ of curing agent). Commercial $8.5 \times 15 \mathrm{~cm}$ Teflon ${ }^{\circledR}$ oven dishes were initially cleaned using acetone, IPA and deionized water followed by a dehydration bake. Mixtures were then carefully poured into the dishes using a specific volume of the mixture in order to form $1 \mathrm{~mm}$ thick uniform films. Prior to moulding, the preparation involves mixing two liquids of different viscosities $5 \times 10^{-3} \mathrm{~m}^{2} \mathrm{~s}^{-1}$ (vinyl-terminated base) and $1.1 \times 10^{-4} \mathrm{~m}^{2} \mathrm{~s}^{-1}$ (curing agent) which incurs the formation of trapped bubbles. Thus, the samples were finally degassed ( 5 pumping cycles in a lowvacuum chamber) in order to remove the air bubbles formed during mixing. The prepared PDMS mixtures were then placed onto a level hotplate during 2 hours at $100^{\circ} \mathrm{C}$. We refer to this first step heating process as "curing" subsequent heat treatments are refereed to as "post-curing. Note that it was not known exactly how the increase of curing agent could affect the curing process, thus we decided to increase the curing time, compared to data-sheet information (e.g. 45 minutes at $100^{\circ} \mathrm{C}[36]$ ), such that every sample was well cured. Half of each of the resulting PDMS films was then post-cured, in a conventional oven at $165^{\circ} \mathrm{C}$ during 48 hours. The objective of the last curing step is to understand the impact of an upper curing time limit on material stiffness and rupture elongation while simply cured samples constitute our reference base. Finally, flat $4 \mathrm{~mm}$ wide, $60 \mathrm{~mm}$ long and $1 \mathrm{~mm}$ thick tensile bone shaped samples are produced from the PDMS films using a specially made dogbone sample cutter. Figure 1 shows the bone samples and the dimensions and table 1 summarises the set of 45 samples tested un this work, showing mixing ratios, curing times and temperatures.

\begin{tabular}{cccccccc}
\cline { 2 - 7 } & \multicolumn{8}{c}{ mixing ratios (Base by weight, Agent by weight) } \\
& $19: 1$ & $10: 1$ & $9: 1$ & $8: 1$ & $7: 1$ & $4: 1$ & $2: 1$ \\
\hline $2 \mathrm{~h} 100^{\circ} \mathrm{C}$ & 2 & 3 & 3 & 2 & 2 & 3 & 3 \\
$48 \mathrm{~h} 165^{\circ} \mathrm{C}$ & 4 & 4 & 4 & 4 & 4 & 5 & 2 \\
\hline
\end{tabular}

Table 1: Summary of the 45 tested PDMS samples as a function of the fabrication process: cured $=2 \mathrm{~h} 100^{\circ} \mathrm{C}$, post-cured $=48 \mathrm{~h}$ $165^{\circ} \mathrm{C}$

\subsection{Measurement methods}

We will focus, in the mechanical measurements part of this work, on the measurement of the initial tangent (elastic) modulus, the Poisson's ratio and the rupture elongation. To achieve such a mechanical investigation, increasing cyclic tests has been preferred to monotonic tensile ones since they allow one to identify material hysteresis due, in part, to viscosity [37]. This is crucial complementary information towards an understanding of how manufacturing processes affect the sample's mechanical behavior.
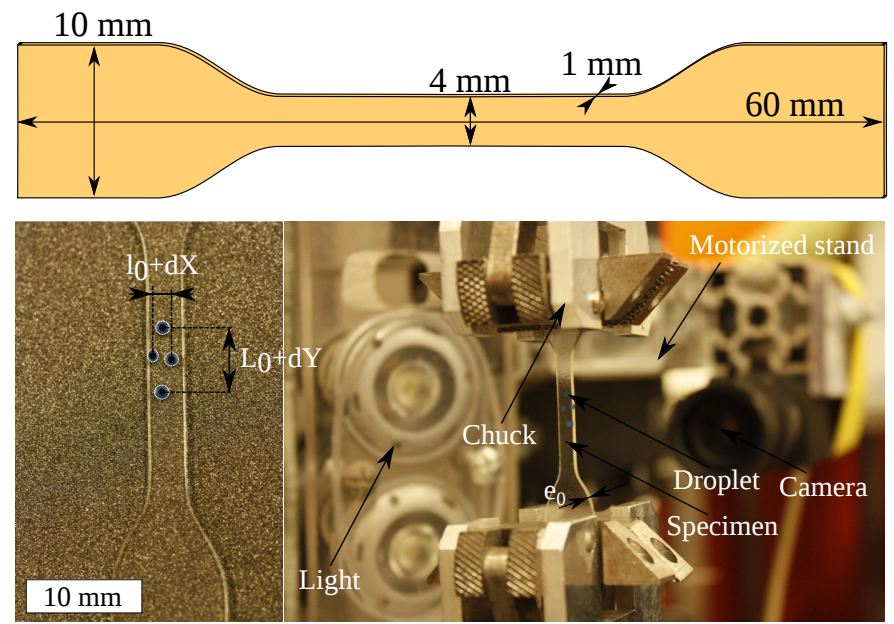

Figure 1: Experimental setup using flat $4 \mathrm{~mm}$ wide, $60 \mathrm{~mm}$ long and $1 \mathrm{~mm}$ thick PDMS tensile test samples. $\Delta X$ and $\Delta Y$ correspond to transversal and axial length variations during test, while $l_{0}, L_{0}$ and $e_{0}$ correspond to the initial width, height and depth respectively.

Thus, PDMS samples have been submitted to increasing cyclic loadings from $0 \mathrm{~N}$ to $5 \%, 10 \%, 15 \%, 20 \%, 30 \%$, $35 \%, 40 \%, 60 \%$ of uniaxial strain, finally up to rupture (see figure 2). Tests were conducted on an Instron 5882 uniaxial testing machine, using a $2 \mathrm{kN}$ cell-force at a constant crosshead speed of $0.5 \mathrm{mms}^{-1}$ which corresponds to an average strain rate close to $10^{-2} \mathrm{~s}^{-1}$ (see figure 2). Notice that the choice of such a strain rate, in the identification of material properties, has been validated by the lack of any hysteresis in the cyclic PDMS behavior (see figure 3). Such cyclic response commonly reveals that the material is not significantly affected by viscosity at such strain rate [37].

Then, we note the nominal stress $\Sigma=\frac{F}{S_{0}}$ with $F$ the force measured by the cell and $S_{0}$ being the initial sample cross-section. The sample thicknesses are measured using a Vernier calliper - this introduces a small measurement uncertainty, of the order of $10^{-1} \mathrm{~mm}$, due to sample stiffness variations; implying an uncertainty on the order of $0.25 \mathrm{~N}$ on the stress estimation.

Strains are measured, within the sample gauge section (see figure 1), by video extensometry. Four painted droplets are put on the sample surface, two for axial displacement and two 


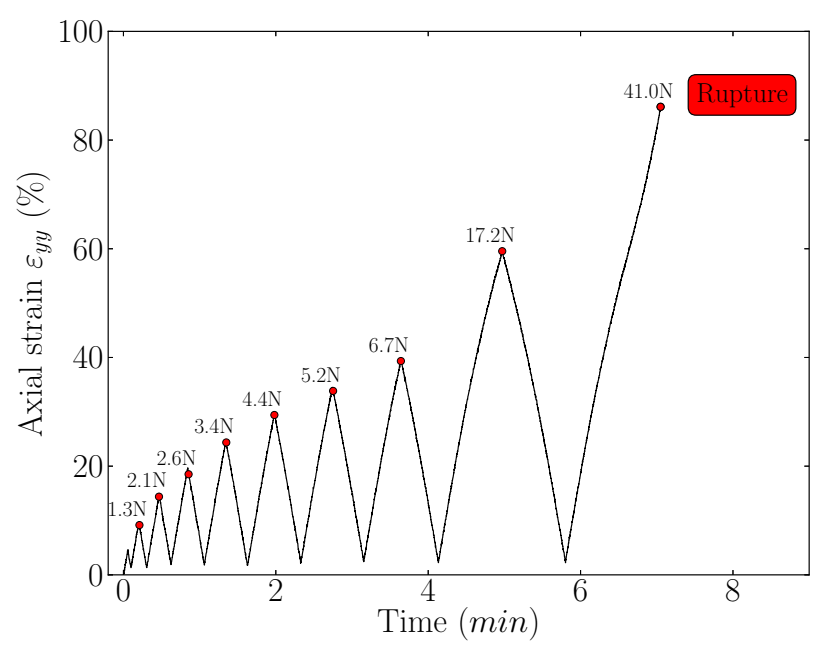

Figure 2: Example of loading cycles applied to tested samples. Force levels correspond to the case of a 10:1 sample.

others for transversal displacement. From the displacement of droplet barycenters, one can compute the axial strain $\varepsilon_{y y}=\frac{\Delta Y}{L_{0}}$, the transversal one $\varepsilon_{x x}=\frac{\Delta X}{l_{0}}$ and the Poisson's ratio $v=-\frac{\varepsilon_{x x}}{\varepsilon_{y y}}$. Notice that although the Poisson's ratio is generally assumed to be constant, near to 0.5 (incompressibility), some research has shown that volume changes occur within elastometric materials during deformation processes and are attributed to damage mechanisms [38, 39, 33, 40] - so the Poisson's ratio decreases with deformation, depending on the damage. Thus, for the sake of clarity we define the initial and the ruptured Poisson's ratios, meaning the undamaged and damage ratio.

As the linear and reversible domain is very different from one sample to another, and the domain size decrease as the material stiffness increases, a systematic and clear definition of the tangent (elastic) modulus is problematic. One should note at this point that, unlike for metals, it is not pertinent to speak of "Young's modulus" in the context of hyperelasticity. One must refer rather to tangent modulus which is defined as the slope of the stress-strain curve which can evolve with strain. Here we define the tangent modulus (E) as the rigidity of the material at low strain. Thus, one can employ the hyperelastic theory and invoke a Mooney-Rivlin model [41, 42] to analytically estimate the initial tangent modulus. We can derive the nominal stress from the generalized Mooney-Rivlin strain energy potential (see equation 1 ).

$$
W=\sum_{i, j=0}^{n} \mathbb{C}_{i j}\left(\mathbb{I}_{1}-3\right)^{i}\left(\mathbb{I}_{2}-3\right)^{j}-\frac{1}{2} k\left(\mathbb{I}_{3}-3\right)^{2}
$$

with $\mathbb{I}_{1}=\lambda_{y}^{2}+\lambda_{x}^{2}+\lambda_{z}^{2}, \mathbb{I}_{2}=\lambda_{y}^{2} \lambda_{x}^{2}+\lambda_{y}^{2} \lambda_{z}^{2}+\lambda_{x}^{2} \lambda_{z}^{2}$ and $\mathbb{I}_{3}=\operatorname{det} \mathbb{F}$ the strain invariants, $(x ; y ; z)$ the transversal, the axial and the orthogonal space directions respectively, $\mathbb{F}$ is the deformation gradient, $\lambda_{i}$ is the strain ratios, $n$ is the order of the Mooney-
Rivlin model, $k$ is the bulk modulus and $\mathbb{C}_{i j}$ is the hyperelastic constants.

Assuming the uniaxiality of the strains $\left(\lambda_{y}=\lambda, \lambda_{x}=\lambda_{z}=\frac{1}{\sqrt{\lambda}}\right)$ , the incompressibility $\left(\mathbb{I}_{3}=1\right)$, and a $2^{\text {nd }}$ order Mooney-Rivlin model ( $n=2$ (see equation 1 ) ) one obtains :

$$
\begin{aligned}
W= & \mathbb{C}_{10}\left(\mathbb{I}_{1}-3\right)+\mathbb{C}_{01}\left(\mathbb{I}_{2}-3\right)+\mathbb{C}_{20}\left(\mathbb{I}_{1}-3\right)^{2} \\
& +\mathbb{C}_{02}\left(\mathbb{I}_{2}-3\right)^{2}+\mathbb{C}_{11}\left(\mathbb{I}_{1}-3\right)\left(\mathbb{I}_{2}-3\right)
\end{aligned}
$$

One notes $\Sigma=\frac{\partial W}{\partial \lambda}$, so :

$$
\begin{aligned}
\Sigma= & 2\left[\mathbb{C}_{10}\left(\lambda-\frac{1}{\lambda^{2}}\right)+\mathbb{C}_{01}\left(1-\frac{1}{\lambda^{3}}\right)\right. \\
& +2 \mathbb{C}_{20}\left(\lambda-\frac{1}{\lambda^{2}}\right)\left(\lambda^{2}+\frac{2}{\lambda}-3\right) \\
& +2 \mathbb{C}_{02}\left(1-\frac{1}{\lambda^{3}}\right)\left(2 \lambda+\frac{1}{\lambda^{2}}-3\right) \\
& \left.+3 \mathbb{C}_{11}\left[\left(\lambda^{2}-\frac{1}{\lambda^{4}}\right)-\left(\lambda-\frac{1}{\lambda^{2}}\right)-\left(1-\frac{1}{\lambda^{3}}\right)\right]\right]
\end{aligned}
$$

with $\lambda=\varepsilon_{y y}+1$. In equation $3, \Sigma$ is linearly dependant on the following vector $\alpha_{i}=\left\langle\mathbb{C}_{10} \mathbb{C}_{01} \mathbb{C}_{20} \mathbb{C}_{02} \mathbb{C}_{11}\right\rangle$ and the associated shape functions:

$$
N_{i}=2\left(\begin{array}{c}
\lambda-\frac{1}{\lambda^{2}} \\
\left(1-\frac{1}{\lambda^{3}}\right) \\
2\left(\lambda-\frac{1}{\lambda^{2}}\right)\left(\lambda^{2}+\frac{2}{\lambda}-3\right) \\
2\left(1-\frac{1}{\lambda^{3}}\right)\left(2 \lambda+\frac{1}{\lambda^{2}}-3\right) \\
3\left[\left(\lambda^{2}-\frac{1}{\lambda^{4}}\right)-\left(\lambda-\frac{1}{\lambda^{2}}\right)-\left(1-\frac{1}{\lambda^{3}}\right)\right]
\end{array}\right)
$$

Thus the identification of hyperelastic parameters can easily be done through the classical Least Square Method as follows:

$$
\left\{\begin{aligned}
\Phi & =\frac{1}{m} \sum_{k=0}^{m}\left(\Sigma_{\exp }(\lambda)-\alpha_{i} N_{i}(\lambda)\right)^{2} \\
\frac{\partial \Phi}{\partial \alpha_{i}} & =0
\end{aligned}\right.
$$

with $\Phi$ the cost function to minimize, $\Sigma_{\text {exp }}(\lambda)$ the experimental stress value at elongation $\lambda, \Sigma=\alpha_{i} N_{i}(\lambda)$ the modelled stress in Einstein's notation and $m$ the number of experimental data points. Figures 3 shows a stress-strain fit superimposed on the experimental values - this validates the use of the MooneyRivlin potential. Finally, one can simply define the initial (E) 
stiffness as follows:

$$
\begin{aligned}
E= & \lim _{\lambda \rightarrow 1} \frac{\partial \Sigma}{\partial \lambda} \\
= & \lim _{\lambda \rightarrow 1}\left[2 \left[\mathbb{C}_{10}\left(1+\frac{2}{\lambda^{3}}\right)+\frac{3 \mathbb{C}_{01}}{\lambda^{6}}\right.\right. \\
& +2 \mathbb{C}_{20}\left(3 \lambda^{2}-3-\frac{6}{\lambda^{3}}+\frac{6}{\lambda^{4}}\right) \\
& +2 \mathbb{C}_{02}\left(2+\frac{2}{\lambda^{3}}-\frac{9}{\lambda^{4}}+\frac{5}{\lambda^{6}}\right) \\
& \left.\left.+3 \mathbb{C}_{11}\left(2 \lambda-1-\frac{2}{\lambda^{3}}+\frac{4}{\lambda^{5}}-\frac{3}{\lambda^{4}}\right)\right]\right] \\
= & 6 \mathbb{C}_{10}
\end{aligned}
$$

In addition to the mechanical testing, wetting contact angle measurements were performed on the different samples. Deionized water droplets having a typical diameter of $1 \mathrm{~mm}$ were deposited on each sample surface in order to investigate how a change of the material's composition affects the droplet contact angle between PDMS and liquid. The wetting contact angles were measured using a contact angle meter in a humidity/temperature controlled cleanroom environment. These tests have important implications if the materials are used for specific microfluidic applications for example. It is well known that the wetting contact angle of a liquid on PDMS can be modified surface chemistry, e.g. using a plasma [43, 44] and chemical immersion [21]. As we will see we show here that the wetting contact angle of water depends on the bulk mechanical properties of the PDMS - without modification of surface.

\section{Results and Discussion}

\subsection{Mechanical characterization}

According to the PDMS Sylgard ${ }^{\circledR} 184$ datasheet [36] and [26, 28], where a PDMS baking time study has shown that the PDMS stiffness saturates for a curing time over 2 days at $100^{\circ} \mathrm{C}$, one assumes that both curing processes (detailed in section 1.1) constitute a lower and an upper limit of reachable PDMS stiffness. Within these curing limits, some identically made sample have been tested (see table 1) and allow us to present average trends and standard deviations.

Let us first deal with an analysis of the resulting stress-strain curves. Figure 3 presents experimental stress-strain curves obtained on the PDMS samples, including different cross-linker agent concentrations, from 5 to $33 \%$, i.e. ratios of 19:1 to 2:1, and different curing process $-2 \mathrm{~h}$ at $100^{\circ} \mathrm{C}$ (curing) and $48 \mathrm{~h}$ at $160^{\circ} \mathrm{C}$ (post-curing). Additional information is also shown: the rupture strain and the Mooney-Rivlin fit (see equation 4).

Loading strains and stresses reach $95 \%$ and $10 \mathrm{MPa}$ respectively. As already stated, one observes a very large range of behavior: from elastic-linear behavior, on the cured 19:1 sample, to a hyperelastic one - for example on the cured 4:1 sample and even quasi-elastic-linear and "brittle" one (up to $20 \%$ ) on post-cured 2:1 sample. In parallel, one observes a systematic sample hardening from cured (black in figure 3) to

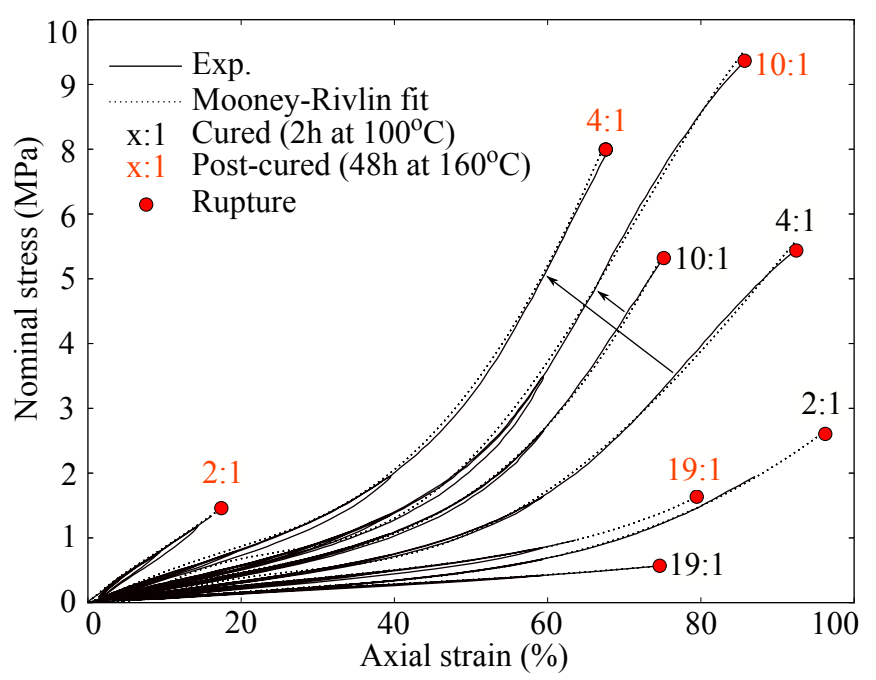

Figure 3: Example of Stress-Strain curves for different mixing ratios $(1: 19,1: 10,1: 4,1: 2)$ and curing processes (cured and post-cured). Mooney-Rivlin fit and rupture strains are superimposed.

post-cured (orange in figure 3) samples. This phenomenon is reflected in a systematic increase of low strain and high strain stiffness and is a function of cross-linker agent concentration. Indeed, one clearly observes how the 19:1 and 10:1 samples slightly harden compared to the $4: 1$ and $2: 1$ ones where curing time and temperature drastically affect the mechanical response. Figure 3 emphases notably the mechanical stability as a function of temperature and the great stretchability of samples at the classic 10:1 mixing ratio.

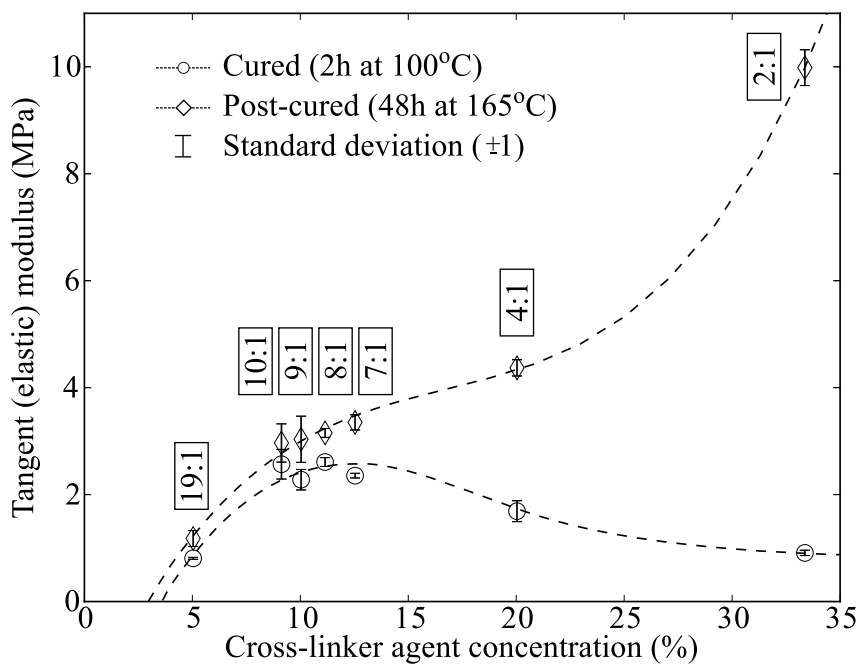

Figure 4: Elastic moduli of PDMS as a function of mixing ratio and curing process: cured $\left(2 \mathrm{~h}\right.$ at $\left.100^{\circ} \mathrm{C}\right)$ and post-cured $(48 \mathrm{~h}$ at $\left.165^{\circ} \mathrm{C}\right)$

Let us now discuss, in a more detailed way, the low strain stiffness (tangent modulus) and rupture limit variations. Figure 


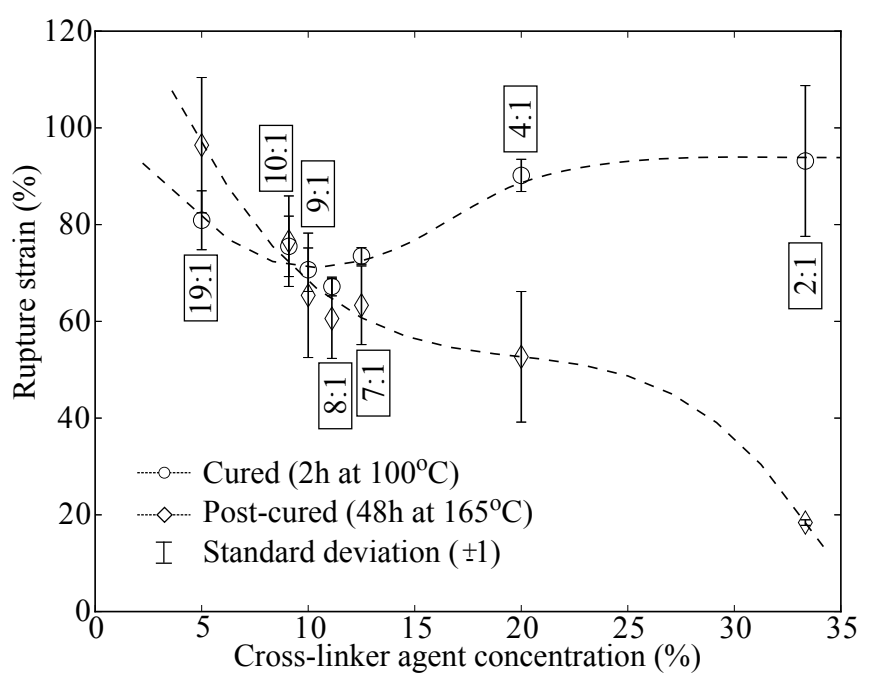

Figure 5: Rupture strains of PDMS as a function of mixing ratio and curing process: cured $\left(2 \mathrm{~h}\right.$ at $\left.100^{\circ} \mathrm{C}\right)$ and post-cured $(48 \mathrm{~h}$ at $\left.165^{\circ} \mathrm{C}\right)$

4 presents the evolution of the tangent (elastic) modulus as a function of cross-linker agent concentration for two curing processes: curing ( $2 \mathrm{~h}$ at $100^{\circ} \mathrm{C}$ : lower bound) and post-curing (48h at $165^{\circ} \mathrm{C}$ : upper bound). Dotted lines are spline interpolations of experimental data sets and error bars are standard deviations $( \pm \sigma)$ obtained by successive tests (see table 1 ). One observes on cured samples $(\mathrm{O})$, that sample stiffness ranges from $800 \mathrm{KPa}$ at $5 \%$ curing agent, to $2.4 \mathrm{MPa}$ at $11 \%$ curing agent and then falls to $900 \mathrm{KPa}$ at $33 \%$ curing agent. An identical trend and levels could be seen in part in [4] where the influence of some mixing ratios $(6: 1,8: 1,10: 1,12: 1)$ on PDMS stiffness have been studied. this confirms, for both baking time and temperatures lower than $8 \mathrm{~h}$ and $150^{\circ} \mathrm{C}$ respectively, the existence of a stiffness maximum for 8:1 materials while other mixing ratios invariably lead to softer materials. The following results (see figure 4) extend such observations from $5 \%$ to $33 \%$ curing agent which is quite interesting with regards to the post-cured behavior. One observes on post-cured samples $(\diamond)$, a quasi-identical trend, slightly stiffer, from 5 to $11 \%$ of curing agent. Then stiffness increase up to $10 \mathrm{MPa}$ at $33 \%$ curing agent - to our knowlegde this is the highest reported elastic modulus in PDMS by combining mixing ratio and curing temperature [28]. Thus, one observes on one hand, that the post-curing process does not significantly affect the PDMS stiffness up to a cross-linker agent concentration near to $10 \%$, and on the other hand, that it reverses the trend over this value. Note on figure 4 the largest difference in stiffness between cured and post-cured occurs in the 2:1 material - thus this mixture enable the user to explore the whole range of stiffnesses using a single mixture over the temperature range study. Thus, the tangent modulus could be increased by a factor higher than 10 by modifying the post-curing time and mixing ratios. Obviously such an increase of the material stiffness drastically affects the rupture strain - let us now discusses this point.
Figure 5 presents the sample rupture strain $\left(\varepsilon_{R}\right)$, defined as the ultimate strain before failure, as a function of cross-linker agent concentration for both curing and post-curing processes. It is well known that the mechanical failure depends primarily on the surface state of the material - especially edge regions where crack initiation sites could be introduced depending on sample cutter quality. This explains notably the increase of scatter in the results shown by the standard deviation error bars. Thus absolute values must be regarded carefully in favour of trend including errors bars. Indeed, as the same cutter tool has been used for the whole set of samples one can assume that results obtained from all samples can be realiably compared and can provide realistic failure trends. Results are clearly in line with previous observations done on PDMS stiffness (see figure 4). If we compare figure 4 and figure 5 we can see that there is a certain symmetry in the results. When the stiffness increases, the rupture strain drops and conversely, when the stiffness decreases the rupture strain rises. Considering cured samples, the rupture strain ranges from $70 \%$ to $110 \%$ with a minimum reached for $8: 1$ samples.

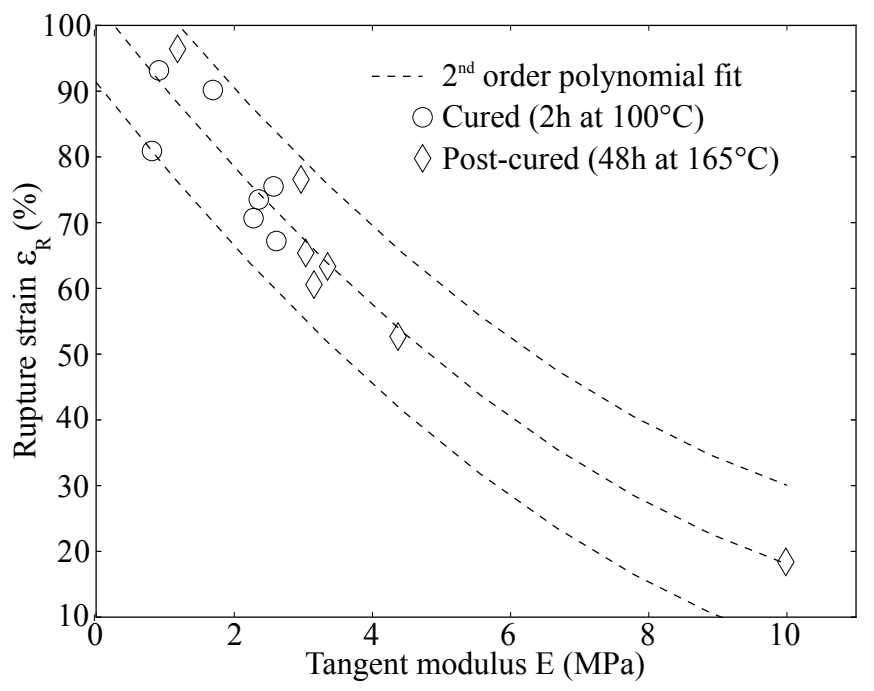

Figure 6: Variation of rupture strain of PDMS samples function of PDMS stiffness. $2^{\text {nd }}$ order polynomial fit equation: $\varepsilon_{R}(E)=103_{ \pm 12}-13.5 E+0.5 E^{2}$

Notice that the same behavior symmetry, between stiffness and rupture, is also observed for post-cured $(\diamond)$ samples see figure 5. For both cured and post-cured samples one can observes that at low cross-linker agent concentration $(<12.5 \%)$ the rupture trends are quite similar. The rupture strain value falls down up to 8:1 samples $\left(\varepsilon_{R} \in[55-70]\right)$. However, when the cross-linker concentration is greater than $12.5 \%$ (up to $33 \%$ ) the rupture strain behavior diverges considerably - see figure 5. Rupture strain of the cured samples (O) increases up to a plateau at $95 \%$ while, for post-cured samples, it falls to 20 $\%$. To complete the analysis of material stiffness and rupture, figure 6 presents the evolution of PDMS rupture as a function of stiffness for both, cured and post-cured samples. A $2^{\text {nd }}$ order polynomial fit is also superimposed and underlines the 
previously observed relationship between, cured or post-cured material stiffness and associated rupture strains. Irrespective of the curing time and temperature (within the limits studied here) one observes that the rupture of samples follows a polynomial digressive law - identified, by Least Square Method, as: $\varepsilon_{R}(E)=103_{ \pm 12}-13.5 E+0.5 E^{2}$. The following relation and figure 6 are particularly interesting for applications where both high rigidity and good flexibility are required since they clearly evidence the limits of such pdms mixtures.

Finally, Figure 7 presents Poisson's ratio of the samples tested as a function of cross-linker concentration for both cured and post-cured samples at both, initial and rupture state.

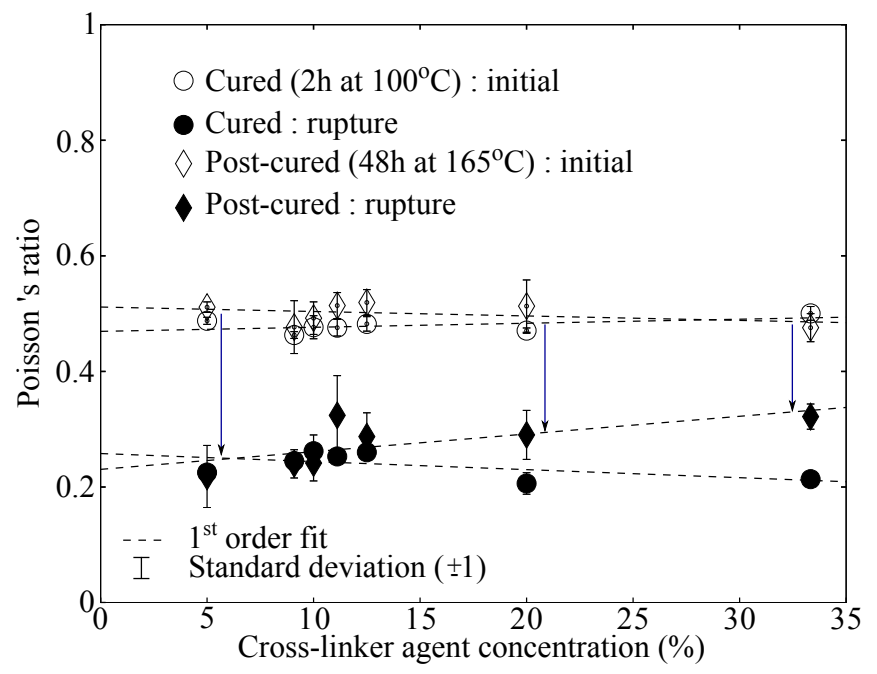

Figure 7: Variation of Poisson's ratio of PDMS samples as a function of cross-linker agent concentration and curing processes (cured and post-cured) at initial and rupture state.

One observes that the initial Poisson's ratio remains almost unchanged whatever the curing process and mixing ratio with a mean value of $0.5 \pm 0.05$ as classically shown in the literature. Secondly, one observes a fall of the Poisson's ratio up to rupture. Table 2 presents, with more details, the change of the Poisson's ratio (in \%).

\begin{tabular}{cccccccc} 
& \multicolumn{9}{c}{ mixing ratios (Base by weight, Agent by weight) } \\
& $19: 1$ & $10: 1$ & $9: 1$ & $8: 1$ & $7: 1$ & $4: 1$ & $2: 1$ \\
\hline $2 \mathrm{~h} 100^{\circ} \mathrm{C}$ & -54 & -47 & -45 & -47 & -46 & -56 & -57 \\
$48 \mathrm{~h} 165^{\circ} \mathrm{C}$ & -57 & -50 & -51 & -37 & -45 & -44 & -32 \\
\hline
\end{tabular}

Table 2: Drop of Poisson's ratio from initial to rupture state (in $\%)$

The results are quite similar for all samples, with a mean value of $-48 \%$ and a standard deviation of $7 \%$, and its physical origin is probably the material cavitation leading to volume changes [40]. Nevertheless, one observes that post-cured samples are systematically less sensible to this phenomenon. According to the fact that these materials are stiffer, especially the 4:1 (Cross-linker: $20 \%$ ) and 2:1 (Cross-linker: $33 \%$ ) ratios, one could speculate that the increase of material rigidity limits the cavitation phenomenon.

\subsection{Wetting contact angle}

Table 3 and figure 8 presents results of wetting contact angle analysis done on PDMS samples as well as experimental pictures of droplets in both, lower and higher, wetting conditions.

\begin{tabular}{ccccccc}
\cline { 2 - 7 } & \multicolumn{5}{c}{ mixing ratios (Base by weight, Agent by weight) } \\
& $19: 1$ & $10: 1$ & $9: 1$ & $8: 1$ & $7: 1$ & $4: 1$ \\
\hline 2h $100^{\circ} \mathrm{C}$ & 109.7 & 107.25 & 100.8 & 105.65 & 105.15 & 111.6 \\
& \pm 0.85 & \pm 0.78 & \pm 1.13 & \pm 0.07 & \pm 0.92 & \pm 0.57 \\
48h $165^{\circ} \mathrm{C}$ & 109.6 & 108.2 & 105.05 & 112.55 & 105.5 & 96.75 \\
& \pm 0 & \pm 1.13 & \pm 0.92 & \pm 2.05 & \pm 0.28 & \pm 0.35 \\
\hline
\end{tabular}

Table 3: Mean value $\left({ }^{\circ}\right)$ and standard deviation of wetting contact angle between deionized water droplets and PDMS surfaces.

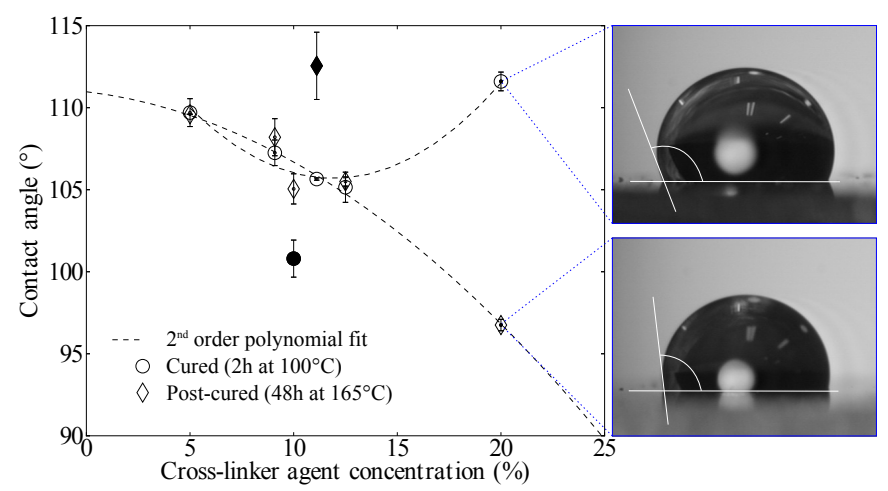

Figure 8: Variation of wetting contact angle between deionized water droplets and PDMS surface function of cross-linker concentration for both, curing and post-curing, process.

Black markers within Figure 8 are experimental points which deviate significantly from the trend - they are consequently not taking into account in the $2^{\text {nd }}$ order polynomial fit presented in figure 8 by dotted lines.

Thus, by not considering these points one can see a relationship between cross-linker agent concentration and surface contact angle on both, cured (O) and post-cured samples $(\diamond)$. Considering first, post-cured samples, one observes a monotonic trend. Indeed, when cross-linker agent concentration increases, and thus material stiffness increases too, one observes a decrease of the wetting contact angle from $109.6^{\circ}$ to $96.75^{\circ}$ (see table 3), i.e. a decrease of $12 \%$. Considering cured samples (O), one observes an identical trend from 5 to $12.5 \%$ of cross-linker agent concentration, meaning from 19:1 to $7: 1$ mixing ratios. Nevertheless, over $12.5 \%$ of cross-linker agent concentration the wetting contact angle increases to $111.6^{\circ}$. A previous study [45] considering mixing ratios from 50:1 to 10:1 has demonstrated that the wetting contact angle changes very little - in good agreement with our results (see figure 8) for all samples (cured and post-cured) having a cross-linker 
agent concentration lower than 9\% (PDMS 10:1). On the other hand, it is also shown in [21] that the wetting contact angle changes very little considering mixing ratios from 19:1 to near $1: 1$. These results are explanable with reference to the figure 4 - in the absence of high temperature curing step, the stiffness remains virtualy the same and relatively small over the whole range of mixtures, whereas a high temperature curing step leads to a significant increase of the stiffness for cross-linker agent concentration over $12.5 \%$ (PDMS 7:1). Our work here implies that in order to observe a significant change in the wetting contact angle, mixing ratios from 10:1 to 2:1 - and even greater - are necessary in addition to a high temperature/long time curing step.

Considering our results it is interesting to notice that such an increase of contact angle is totally in keeping with the fall of material stiffness (cured samples) between 7:1 and 4:1 mixing ratios observed on figure 4. Secondly, the occurrence of a contact angle deviation between cured and post-cured samples is also in line with observation done on material stiffness. Indeed, over $12.5 \%$ of cross-linker agent concentration (PDMS $7: 1)$ the stiffness of cured samples (O) falls while the contact angle rises and inversely, the stiffness of post-cured samples $(\diamond)$ rises while the contact angle drops. Such comparisons validate contact angle observations and allow the identifying of a relationship between PDMS stiffness and the material's wetting contact angle.

Figure 9 shows the evolution of wetting contact angle of deionized water as a function of the measured PDMS stiffness on both cured and post-cured samples. Irrespective of the curing process, the observed trend is that the wetting contact angle of the water - reduces with increasing material stiffness, i.e. it would appear that the surface energy of the material increases with increasing stiffness. Then, as already observed in figure 8 , the figure 9 allows one to define a relationship between both quantities in the form of a $2^{\text {nd }}$ order polynomial function such as: $\theta(E)=108.5_{ \pm 3}+2.6 E-1.2 E^{2}$. The figure demonstrates that the contact angle on both, cured and post-cured samples, follows an identical law governed by the material stiffness.

The following results can be understood by analogy with the process of PDMS surface oxidation during oxygen plasma exposure. Indeed it is well known that a significant reduction of wetting contact angle on PDMS surfaces can be achieved by exposing the PDMS to oxygen plasma, e.g. [43]. It is also well known that such an exposure results in a chemical change of the PDMS surface whose macroscopic manifestation is the creation of a thin and stiff silica-like film with a stiffness about $70 \mathrm{GPa}$ [46]. Thus, from a mechanical point of view, hardening the PDMS crust and increasing its surface energy reduces the wetting contact angle. In consequence, oxygen plasma treatment is a way to drastically but temporarily [47] affect the PDMS wettability by considerably modifying the surface stiffness. Here we show that the wetting contact angle can be modified by tuning the volume stiffness of the PDMS. The effect is less than in the case of an oxygen plasma treatment [43] but more durable. Such result is obviously interesting concerning PDMS in microfluidic applications.

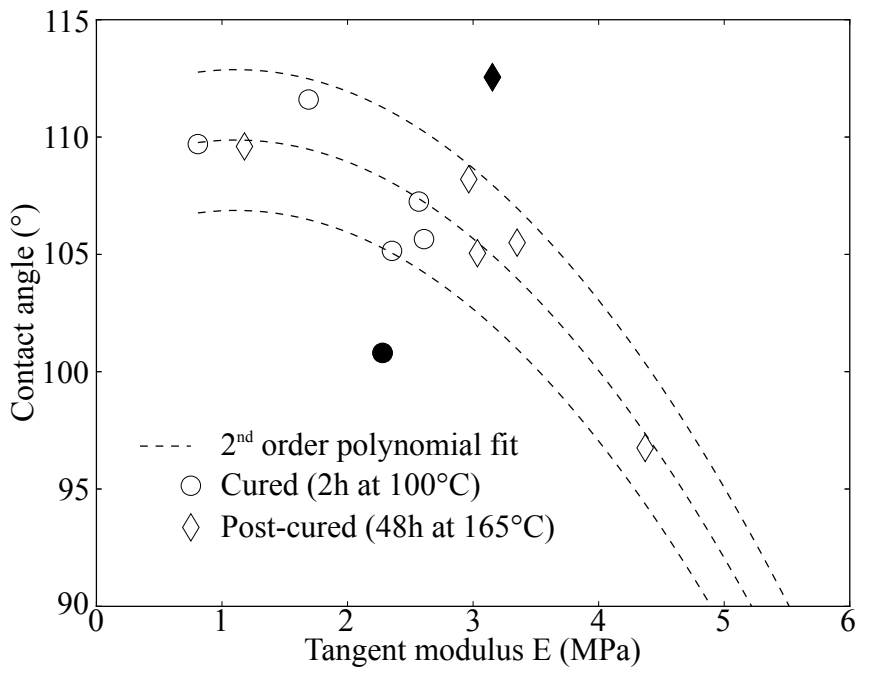

Figure 9: Variation of the wetting contact angle $(\theta)$ between deionized water droplets and PDMS surface as a function of PDMS stiffness $(E)$. $2^{\text {nd }}$ order polynomial fit equation: $\theta(E)=108.5_{ \pm 3}+2.6 E-1.2 E^{2}$

\section{Conclusions}

This work demonstrates that by modifying the cross-linker agent concentration (mixing ratio), the curing time and the curing temperature, one can tune the stiffness of $1 \mathrm{~mm}$ thick PDMS samples from $800 \mathrm{kPa}$ to $10 \mathrm{MPa}$ - this is the largest stiffness range reported to date. A set of mixing ratios from 19:1 to $2: 1$ and two curing processes, i.e. $2 \mathrm{~h}$ at $100^{\circ} \mathrm{C}$ and $48 \mathrm{~h}$ at $165^{\circ} \mathrm{C}$, has been investigated and no problems associated with moulding have been observed. A great diversity of mechanical responses has been observed, from linear- to hyper-elastic including a "brittle" behavior in the stiffest materials (2:1). Indeed, from our results at two extreme curing processes we can speculate that the 2:1 ratio, combined with the appropiate curing process, enables the user to explore the whole stiffness range from $800 \mathrm{kPa}$ to $10 \mathrm{MPa}$. The relationship between rupture strain and material stiffness as well as between wetting contact angle and material stiffness has been systematically and statistically investigated. Our results indicate that both the rupture strain and the wetting contact angle (i.e. the surface energy of the PDMS) evolve as the square of material stiffness. We believe that by increasing the range of mechanical properties of PDMS we render the material more compatible with technological processes, e.g. depositon of materials such as metals, and at the same time maintaining its flexibility for specific applications.

\section{Acknowledgements}

The authors would like to thanks Dr E. Charkaluk from the Laboratory of Mechanics of Lille for helpful discussions and the access to laboratory facilities. 
Financial support is acknowledged from the EQUIPEX project "LEAF" (ANR-11-EQPX-0025) funded by the French government.

\section{References}

[1] C. Friedel, J. Crafts, Liebigs Ann. Chem 12731.

[2] S. P. Lacour, D. Chan, S. Wagner, T. Li, Z. Suo, Mechanisms of reversible stretchability of thin metal films on elastomeric substrates, Applied Physics Letters 88 (20) (2006) 204103-204103.

[3] K. S. Kim, Y. Zhao, H. Jang, S. Y. Lee, J. M. Kim, K. S. Kim, J.-H. Ahn, P. Kim, J.-Y. Choi, B. H. Hong, Large-scale pattern growth of graphene films for stretchable transparent electrodes, Nature 457 (7230) (2009) 706-710.

[4] H. Hocheng, C.-M. Chen, Y.-C. Chou, C.-H. Lin, Study of novel electrical routing and integrated packaging on bio-compatible flexible substrates, Microsystem Technologies 16 (3) (2010) 423-430. doi:10. 1007/s00542-009-0930-2

[5] A. Nathan, A. Ahnood, M. T. Cole, S. Lee, Y. Suzuki, P. Hiralal, F. Bonaccorso, T. Hasan, L. Garcia-Gancedo, A. Dyadyusha, et al., Flexible electronics: the next ubiquitous platform, Proceedings of the IEEE 100 (Special Centennial Issue) (2012) 1486-1517.

[6] A. Romeo, Q. Liu, Z. Suo, S. Lacour, Elastomeric substrates with embedded stiff platforms for stretchable electronics, Applied Physics Letters 102 (13) (2013) 131904. doi:http://dx.doi.org/10.1063/1. 4799653

[7] A. Kumar, G. M. Whitesides, Features of gold having micrometer to centimeter dimensions can be formed through a combination of stamping with an elastomeric stamp and an alkanethiol ink followed by chemical etching, Applied Physics Letters 63 (14) (1993) 2002-2004.

[8] R. J. Jackman, J. L. Wilbur, G. M. Whitesides, Fabrication of submicrometer features on curved substrates by microcontact printing, Science 269 (5224) 664-666.

[9] Y. Xia, G. M. Whitesides, Soft lithography, Annual review of materials science 28 (1) (1998) 153-184.

[10] H. Schmid, B. Michel, Siloxane polymers for high-resolution, highaccuracy soft lithography, Macromolecules 33 (8) (2000) 3042-3049. doi: $10.1021 / \mathrm{ma} 9820341$

[11] K. M. Choi, J. A. Rogers, A photocurable poly (dimethylsiloxane) chemistry designed for soft lithographic molding and printing in the nanometer regime, Journal of the American Chemical Society 125 (14) (2003) 4060 4061. doi: 10.1002/adfm. 200600710

[12] D. R. Barbero, M. S. Saifullah, P. Hoffmann, H. J. Mathieu, D. Anderson, G. A. Jones, M. E. Welland, U. Steiner, High-resolution nanoimprinting with a robust and reusable polymer mold, Advanced Functional Materials 17 (14) (2007) 2419-2425. doi:10.1002/adfm. 200600710

[13] P. Jothimuthu, A. Carroll, A. A. S. Bhagat, G. Lin, J. E. Mark, I. Papautsky, Photodefinable pdms thin films for microfabrication applications, Journal of Micromechanics and Microengineering 19 (4) (2009) 045024. doi:10.1088/0960-1317/19/4/045024

[14] Y.-T. Hsieh, H. Hsieh, Y.-C. Lee, A soft photo-mask with embedded carbon black and its application in contact photolithography, Journal of Micromechanics and Microengineering 24 (8) (2014) 085006.

[15] H. Takao, K. Miyamura, H. Ebi, M. Ashiki, K. Sawada, M. Ishida, A mems microvalve with pdms diaphragm and two-chamber configuration of thermo-pneumatic actuator for integrated blood test system on silicon, Sensors and Actuators A: Physical 119 (2) (2005) 468-475.

[16] F. Schneider, T. Fellner, J. Wilde, U. Wallrabe, Mechanical properties of silicones for mems, Journal of Micromechanics and Microengineering 18 (6) (2008) 065008.

[17] F. Schneider, J. Draheim, R. Kamberger, U. Wallrabe, Process and material properties of polydimethylsiloxane (pdms) for optical mems, Sensors and Actuators A: Physical 151 (2) (2009) 95-99.

[18] S. H. Cho, H. M. Andersson, S. R. White, N. R. Sottos, P. V. Braun, Polydimethylsiloxane-based self-healing materials, Advanced Materials 18 (8) (2006) 997-1000.

[19] B.-H. Jo, L. M. Van Lerberghe, K. M. Motsegood, D. J. Beebe, Threedimensional micro-channel fabrication in polydimethylsiloxane (pdms) elastomer, Journal of Microelectromechanical Systems 9 (1) (2000) 7681.
[20] D. T. Eddington, R. H. Liu, J. S. Moore, D. J. Beebe, An organic selfregulating microfluidic system, Lab on a Chip 1 (2) (2001) 96-99.

[21] A. Mata, A. J. Fleischman, S. Roy, Characterization of polydimethylsiloxane (pdms) properties for biomedical micro/nanosystems, Biomedical microdevices 7 (4) (2005) 281-293.

[22] A. A. S. Bhagat, P. Jothimuthu, I. Papautsky, Photodefinable polydimethylsiloxane (pdms) for rapid lab-on-a-chip prototyping, Lab on a Chip 7 (9) (2007) 1192-1197. doi:10.1039/B704946C

[23] B.-Y. Kim, L.-Y. Hong, Y.-M. Chung, D.-P. Kim, C.-S. Lee, Solventresistant pdms microfluidic devices with hybrid inorganic/organic polymer coatings, Advanced Functional Materials 19 (23) (2009) 3796-3803. doi:10.1002/adfm.200901024

[24] J. Lötters, W. Olthuis, P. Veltink, P. Bergveld, The mechanical properties of the rubber elastic polymer polydimethylsiloxane for sensor applications, Journal of Micromechanics and Microengineering 7 (3) (1997) 145.

[25] S. L. Peterson, A. McDonald, P. L. Gourley, D. Y. Sasaki, Poly(dimethylsiloxane) thin films as biocompatible coatings for microfluidics devices: Cell culture and flow studies with glial cells, Journal of Biomedical Materials Research Part A 72A (1) (2005) 10-18. doi: 10.1002/jbm.a.30166

[26] D. T. Eddington, W. C. Crone, D. J. Beebe, Development of process protocols to fine tune polydimethylsiloxane material properties, in: 7th International Conference on Miniaturized Chemical and Biochemical Analysis Systems, Squaw Valley, California, USA, 2003, pp. 1089-1092.

[27] E. A. Wilder, S. Guo, S. Lin-Gibson, M. J. Fasolka, C. M. Stafford, Measuring the modulus of soft polymer networks via a buckling-based metrology, Macromolecules 39 (12) (2006) 4138-4143. doi:10.1021/ ma060266b

[28] D. Fuard, T. Tzvetkova-Chevolleau, S. Decossas, P. Tracqui, P. Schiavone, Optimization of poly-di-methyl-siloxane (pdms) substrates for studying cellular adhesion and motility, Microelectronic Engineering 85 (5) (2008) 1289-1293. doi:10.1016/j.mee.2008.02.004

[29] K. Khanafer, A. Duprey, M. Schlicht, R. Berguer, Effects of strain rate, mixing ratio, and stress-strain definition on the mechanical behavior of the polydimethylsiloxane (pdms) material as related to its biological applications, Biomedical microdevices 11 (2) (2009) 503-508. doi:10.1007/s 10544-008-9256-6

[30] M. Liu, J. Sun, Y. Sun, C. Bock, Q. Chen, Thickness-dependent mechanical properties of polydimethylsiloxane membranes, Journal of Micromechanics and Microengineering 19 (3) (2009) 035028.

[31] Q. Cheng, Z. Sun, G. A. Meininger, M. Almasri, Note: Mechanical study of micromachined polydimethylsiloxane elastic microposts, Review of Scientific Instruments 81 (10) (2010) 106104.

[32] Z. Wang, Polydimethylsiloxane mechanical properties measured by macroscopic compression and nanoindentation techniques, Ph.D. thesis, University of South Florida (2011).

[33] J. De Crevoisier, G. Besnard, Y. Merckel, H. Zhang, F. Vion-Loisel, J. Caillard, D. Berghezan, C. Creton, J. Diani, M. Brieu, et al., Volume changes in a filled elastomer studied via digital image correlation, Polymer Testing 31 (5) (2012) 663-670.

[34] K. Yoo, Y. Takei, S. Kim, S. Chiashi, S. Maruyama, K. Matsumoto, I. Shimoyama, Direct physical exfoliation of few-layer graphene from graphite grown on a nickel foil using polydimethylsiloxane with tunable elasticity and adhesion, Nanotechnology 24 (20) (2013) 205302.

[35] I. D. Johnston, D. K. McCluskey, C. K. L. Tan, M. C. Tracey, Mechanical characterization of bulk sylgard 184 for microfluidics and microengineering Journal of Micromechanics and Microengineering 24 (3) (2014) 035017 . URL http://stacks . iop.org/0960-1317/24/i=3/a=035017

[36] D. Corning, Sylgard $\mathbb{R} 184$ silicone elastomer kit web page, http://www.dowcorning.com/applications/search/ products /Details .aspx?prod=01064291

[37] M. Brieu, J. Diani, Material directional based constitutive law for mullins induced anisotropy, in: Constitutive models for rubbe-proceedings-, Vol. 4, Balkema, 2005, p. 257.

[38] H. Jones, H. Yiengst, Dilatometer studies of pigment-rubber systems, Rubber Chemistry and Technology 14 (1) (1941) 113-124.

[39] J. Ramier, L. Chazeau, C. Gauthier, L. Stelandre, L. Guy, E. PeuvrelDisdier, In situ sals and volume variation measurements during deformation of treated silica filled sbr, Journal of materials science 42 (19) (2007) 
8130-8138.

[40] Y. Merckel, J. Diani, M. Brieu, J. Caillard, Effects of the amount of fillers and of the crosslink density on the mechanical behavior of carbonblack filled styrene butadiene rubbers, Journal of Applied Polymer Science 129 (4) (2013) 2086-2091.

[41] M. Mooney, A theory of large elastic deformation, Journal of applied physics 11 (9) (1940) 582-592.

[42] R. W. Ogden, Non-linear elastic deformations, Courier Dover Publications, 1997.

[43] S. Bhattacharya, A. Datta, J. M. Berg, S. Gangopadhyay, Studies on surface wettability of poly (dimethyl) siloxane (pdms) and glass under oxygen-plasma treatment and correlation with bond strength, Microelectromechanical Systems, Journal of 14 (3) (2005) 590-597.

[44] K. Tsougeni, A. Tserepi, G. Boulousis, V. Constantoudis, E. Gogolides, Control of nanotexture and wetting properties of polydimethylsiloxane from very hydrophobic to super-hydrophobic by plasma processing, Plasma Processes and Polymers 4 (4) (2007) 398-405.

[45] X. Q. Brown, K. Ookawa, J. Y. Wong, Evaluation of polydimethylsiloxane scaffolds with physiologically-relevant elastic moduli: interplay of substrate mechanics and surface chemistry effects on vascular smooth muscle cell response, Biomaterials 26 (16) (2005) 3123-3129.

[46] F. A. Bayley, J. L. Liao, P. N. Stavrinou, A. Chiche, J. T. Cabral, Wavefront kinetics of plasma oxidation of polydimethylsiloxane: limits for sub- $\mu$ m wrinkling, Soft matter 10 (8) (2014) 1155-1166.

[47] M. Morra, E. Occhiello, R. Marola, F. Garbassi, P. Humphrey, D. Johnson, On the aging of oxygen plasma-treated polydimethylsiloxane surfaces, Journal of Colloid and Interface Science 137 (1) (1990) 11-24.

\section{Biographies}

Rian Seghir was born in Marseilles (France). He received a Ph.D. in Mechanical Engineering from the Ecole Centrale de Lille in 2013. Following a postdoctoral position at the Laboratoire de Mécanique de Lille (LML), he is now a CNRS Postdoctoral fellow working at the Institut d'Electronique, de Microélectronique et de Nanotechnologie (IEMN) at the University of Lille. His research interests include flexible electronics and systems, electro-mechanical properties of thin films, optical metrology and digital image correlation.

Steve Arscott was born in Plymouth (UK). He obtained his Ph.D. from the University of Manchester (UK) in 1994. Following postdoctoral positions at the University of Leeds (UK) and the University of Lille (France) at the Institut d'Electronique, de Microélectronique et de Nanotechnologie (IEMN), he joined the Centre National de la Recherche Scientifque (CNRS) as a research scientist based at IEMN. His current research interests include micro/nanotechnology, micro/nanoelectromechanical systems, micro/nanofluidics, micro energy sources, flexible electronics and systems, electrospraying and electrowetting. 